\title{
NUEVOS REGISTROS DE LONGITUD Y DIETA DE Micrurus mipartitus (DUMÉRIL, BIBRON Y DUMÉRIL, 1854) (SERPENTES: ELAPIDAE).
}

\section{New records of length and diet of Micrurus mipartitus (Duméril, Bibron and Duméril, 1854) (Serpentes: Elapidae).}

Luis Enrique Vera-Pérez', Jorge Alberto Zúñiga-Baos², Santiago Ayerbe González ${ }^{3}$

1. Investigador independiente, Carrera 8 \# 2A-12 Barrio Las 2uintas, La Plata (Huila).luchoveraperez@gmail.com

2. Investigador independiente. jorzuba@gmail.com

3. Grupo de Investigaciones Herpetológicas y Toxinológicas Universidad del Cauca.sayerbe@hotmail.com.

\section{Resumen}

La "coral rabo de ají" Micrurus mipartitus se distribuye en Centroamérica y Sudamérica, y es referenciada como una serpiente delgada que usualmente mide entre $60-80 \mathrm{~cm}$ pero que registra una longitud máxima de $120 \mathrm{~cm}$. Con respecto a la dieta, se ha documentado que en su hábitat natural se alimenta de algunas especies de serpientes, lagartos, anfisbénidos, anuros y cecilias, mientras que animales en cautiverio generalmente se rehúsan a alimentarse de manera voluntaria haciendo difícil el mantenimiento. Con base en dos individuos capturados en el departamento del Huila, Colombia y del proceso de mantenimiento en cautiverio en el Centro de Investigaciones Biomédicas de la Universidad del Cauca (CIBUC), se presentan dos nuevos registros que aumentan los valores conocidos de la longitud máxima de $M$. mipartitus, así como nuevos componentes de su dieta.

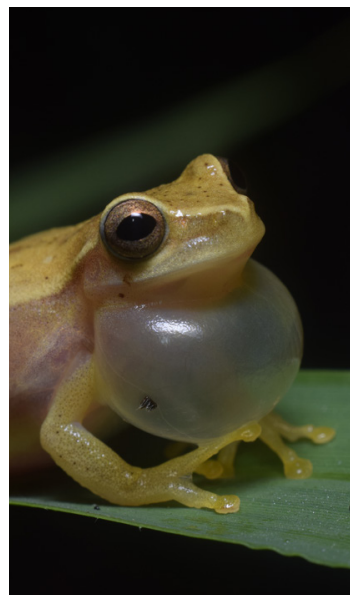

Historia del artículo

Fecha de recepción :

$29 / 03 / 2019$

Fecha de aceptación:

$04 / 08 / 2018$ 
Palabras claves: Cauca, Huila, Colombia, coral rabo de ají, longitud máxima.

\section{Abstract}

The "red-tailed coral snake" Micrurus mipartitus is distributed in Central and South America, and it is referenced as a thin snake that usually measures between $60-80 \mathrm{~cm}$ but registers a maximum length of $120 \mathrm{~cm}$. Regarding its diet, some species of snakes, lizards, amphisbaenians, anurans and caecilians have been documented as its preys on the wild, while captive animals generally refuse to eat voluntarily making it difficult to maintain. Based on two individuals captured in the department of Huila, Colombia, and the process of maintenance in captivity at the Centro de Investigaciones Biomédicas of the Universidad del Cauca (CIBUC), we present two new records that increase the known values of the maximum length of $M$. mipartitus, as well as new components of its diet.

Key words: Cauca, Huila, Colombia, red-tailed coral snake, maximum length.

\section{Introducción}

El género Micrurus contiene actualmente 81 especies reconocidas (Uetz et al., 2019), distribuidas desde el sur de los Estados Unidos hasta el norte de Argentina (Roze, 1996; Campbell y Lamar, 2004). En Colombia este género está representado por 30 especies (Uetz et al., 2019), de las cuales, Micrurus mipartitus es una de las más comunes y se encuentra ampliamente distribuida en el territorio nacional con registros en 22 departamentos: Antioquia,
Atlántico, Boyacá, Caldas, Casanare, Cauca (incluida la isla Gorgona), Cesar, Chocó, Córdoba, Cundinamarca, Huila, La Guajira, Magdalena, Meta, Nariño, Norte de Santander, Putumayo, Quindío, Risaralda, Santander, Tolima y Valle del Cauca (Wallach et al., 2014; Ríos-Soto et al., 2018). Su distribución va desde el nivel del mar hasta 2.750 msnm (Pérez-Santos y Moreno, 1988). Actualmente se reconocen cinco subespecies de M. mipartitus: M. m. mipartitus, M. m. anomalus, M. m. decussatus, M. m. popayanensis y M. m. rozei; de las cuales las cuatro primeras están presentes en Colombia (Cambell y Lamar, 2004).

Micrurus mipartitus es una serpiente de coral largay delgada caracterizada principalmente por tener una cabeza negra no diferenciada del cuerpo con una banda supracefálica naranja-rojiza. Además, presenta 34-84 anillos negros en el cuerpo (generalmente completos) alternados con anillos claros que varían entre blanco, crema, crema-verdoso y amarillo; y uno a nueve anillos negros en la cola alternados por anillos del mismo color de la banda supracefálica (Ayerbe et al., 1990, Roze, 1996; Campbell y Lamar, 2004).

Todos los autores anteriormente mencionados han generado un conocimiento integral de los aspectos biológicos y ecológicos de esta especie, aunque algunos aspectos de estos son referidos de manera general de acuerdo al conocimiento de todo el género. Roze (1996) plantea que uno de los interrogantes más frecuentes sobre las corales es ¿cuán grandes son?, y resuelve que la respuesta no es sencilla, pues para ello es necesario una muestra grande que 
sea representativa y permita determinar el tamaño máximo de una especie. Además, uno de los principales problemas al tratar de hallar la longitud de una serpiente es la limitación para obtener medidas con el animal vivo o recientemente muerto, pues la mayoría de ejemplares disponibles están preservados y su tamaño disminuye, así se emplee el método de colocar una cuerda sobre la superficie dorsal o ventral, retirarla y tomar la medida señalada (Roze, 1996). Así mismo, la dieta de la mayoría de especies de corales suele relacionarse a modo general por su ofiofagismo y la capacidad de ingerir presas de menor tamaño como lagartos, anfibios ápodos y anfisbénidos.

La longitud estandarizada para adultos de $M$. mipartitus se estimaba entre 50-80 cm y un valor máximo de 100,5 cm (Roze, 1996); pero posteriormente Campbell y Lamar (2004) proponen que esta especie mide usualmente entre $60-80 \mathrm{~cm}$ y que puede alcanzar hasta los $120 \mathrm{~cm}$. Desde entonces, todas las referencias sobre el tamaño de la especie han estado relacionadas con estos valores. Con respecto a su dieta, los registros específicos de presas consumidas en hábitats naturales y en cautiverio incluyen las serpientes Atractus. spp., A. werneri, A. sanctaemartae, Dendrophidion bivittatus, Erythrolamprus epinephelus y Leptophis ahaetulla, el lagarto Lepidoblepharis sanctaemartae, Amphisbaena spp. y la culebra ciega Caecilia guntheri (Ayerbe et al., 1990; Roze, 1996; Ríos-Soto et al., 2018).

\section{Materiales y métodos}

En 2015, durante las labores del manejo de fauna desarrolladas en el Proyecto
Hidroeléctrico El Quimbo se capturaron dos hembras adultas de M. mipartitus anomalus, las cuales se midieron vivas utilizando una regla métrica ajustada en una mesa de disección, luego de introducirlas en tubos de acrílico transparente.

El individuo de mayor tamaño (Fig. 1) fue colectado y medido nuevamente para tener un valor más exacto y se encuentra depositado en la colección herpetológica del Museo de Historia Natural de la Universidad del Cauca (número de campo: LVP 0311), mientras que el segundo individuo se

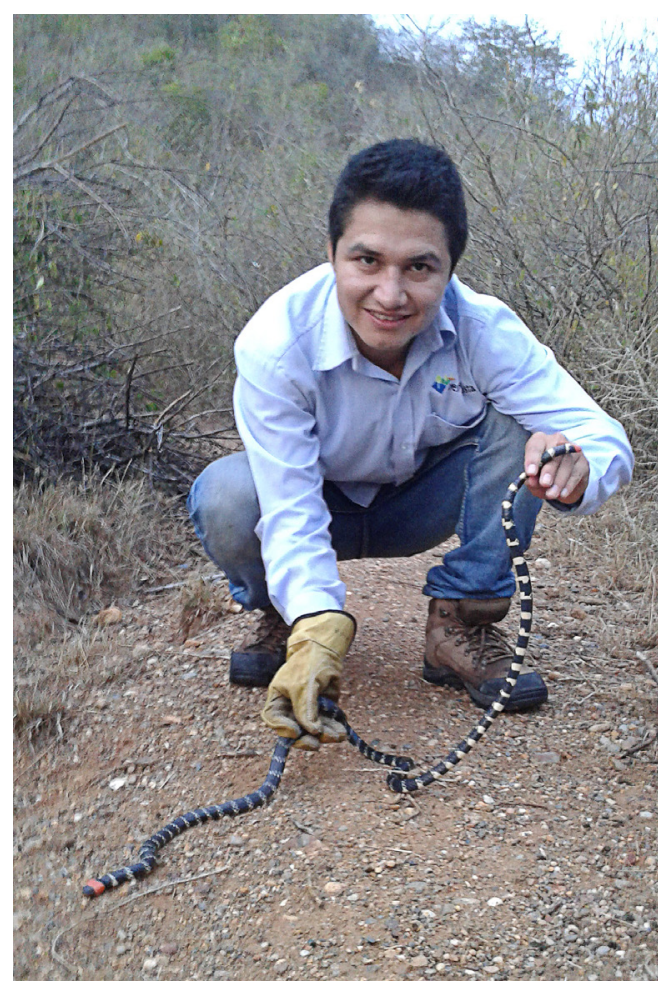

Figura 1. Captura de Micrurus mipartitus anomalus (MHNUG-HE-Se-069o) en el municipio de El Agrado, departamento del Huila, Colombia. 
marcó con elastómeros (código M9O12) y se liberó posteriormente en una zona de restauración ecológica.

Entre el 2010 y 2014, individuos de M. $m$. decussatus y M. m. popayanensis fueron mantenidos en cautiverio en el serpentario del Centro de Investigaciones Biomédicas de la Universidad del Cauca (CIBUC), y durante este periodo se les suministraron presas vivas de diferentes especies de vertebrados, todas capturadas en la ciudad de Popayán. Los resultados obtenidos aportan datos complementarios sobre la longitud máxima y la dieta de la "coral rabo de ají".

\section{Resultados y discusión}

Longitud máxima: el individuo colectado fue capturado el 27 de agosto de 2015 sobre la margen izquierda del río Magdalena, en el Municipio de El Agrado, departamento del Huila, Colombia (2 $2^{\circ} 14^{\prime} 37, \mathrm{O}^{\prime \prime} \mathrm{N} ; 75^{\circ} 40^{\prime} 52,3^{\prime \prime}$ $\mathrm{W} ; 723 \mathrm{msnm}$.) y registró una longitud total de 140,6 cm (longitud rostro-cloaca $=133,5$ $\mathrm{cm}$, longitud caudal $=7,1 \mathrm{~cm})($ Figura 2$)$. El individuo liberado fue capturado el $26 \mathrm{de}$ octubre sobre la margen derecha del río Magdalena, en el Municipio de Gigante, departamento del Huila, Colombia $\left(2^{\circ}\right.$ 22'09,0" N; 75 ${ }^{\circ} 34^{\prime} 17,8$ ” W; 727 msnm) y midió aproximadamente $136 \mathrm{~cm}$ de longitud total, el peso de los dos individuos fue de 227 y $113 \mathrm{~g}$, respectivamente.

Dieta: de las especies ofrecidas como presa en cautiverio, los individuos de $M . m$. decussatus y M. m. popayanensis rechazaron al lagarto Anolis antonii mientras que se alimentaron voluntariamente de Caecilia occidentalis, Pholidobolus vertebralis, Atractus gr. iridescens (Atractus sp. en Ayerbe et al., 1990), A. lehmanni, Dendrophidion bivittatus, Erythrolamprus epinephelus y Sibon nebulatus (Figs. 3 A-G). En todos los eventos de alimentación registrados, las corales realizaron el mismo procedimiento de neutralización y deglución de las presas detallado por Ayerbe et al. (1990), con la adición de que no hay una preferencia en la posición en que éstas son ingeridas (ventral o dorsalmente) y que algunas veces las serpientes se alimentaron sin haber realizado completamente su proceso de ecdisis. Con respecto a su dieta natural, se comprobó el consumo del lagarto Bachia bicolor (Fig. 3H) a través de un individuo regurgitado por un juvenil de M. m. anomalus en el departamento del Huila.

La longitud total de 140,6 cm reportada aquí (MHNUC-HE-Se-0690) representa el máximo registro para Micrurus mipartitus, superando en 20,6 cm la longitud máxima referida por Campbell y Lamar (2004) y posicionando además a la "coral rabo de ají" como una de las especies más largas dentro del género. La longitud del otro individuo referenciado (136 $\mathrm{cm}$ aprox.) muestra que el valor de este nuevo récord puede ser alcanzado por otros individuos al menos en la misma región, y que este no corresponde a un registro excepcional.

Las zonas donde se encontraron estos individuos se caracterizan por ser parches de bosque seco con regeneración temprana que están contiguos a otros tipos de coberturas vegetales como arbustales, 


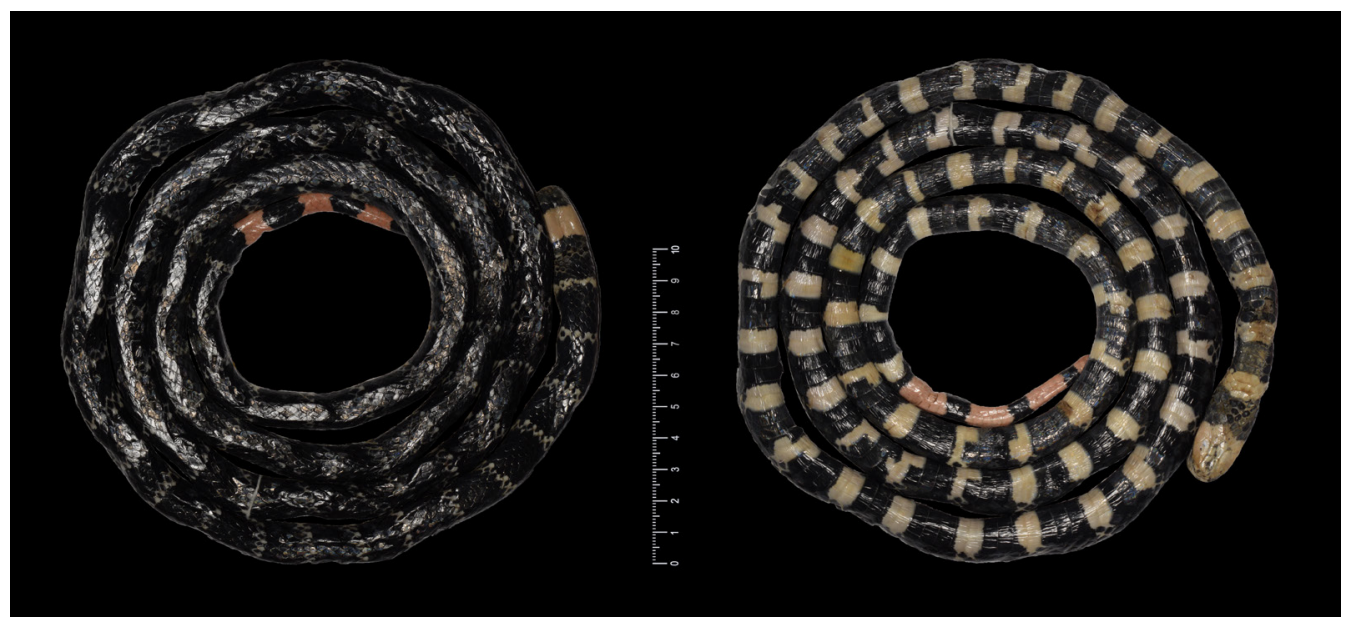

Figura 2. Vista dorsal (izquierda) y ventral (derecha) del nuevo récord de longitud máxima de Micrurus mipartitus (MHNUC-HE-Se-0690).

bosques de ribera y cultivos de cacao además de cuerpos de agua (quebradas La Yaguilga y La Honda). Es su conjunto, estas áreas proporcionan condiciones apropiadas de alta humedad, menor radiación y una gran acumulación de hojarasca, entre otras, que favorecen el refugio, la reproducción y una mayor disponibilidad de especies para depredar y otras que potencialmente podría consumir (ver Moreno-Arias y QuinteroCorzo, 2015). En esta región M. mipartitus es simpátrica con su congénere M. dumerilii, que es más abundante y depreda también al lagarto B. bicolor , además de Leptodeira septentrionalis" e incluso juveniles de su propia especie*. Al estar estrechamente emparentadas y tener similitud en la mayoría de aspectos biológicos y ecológicos, es muy probable que estas dos serpientes

* Registros obtenidos por regurgitación de presas. corales tengan en común más de una especie dentro de su dieta.

Datos sobre la dieta natural de las corales suelen ser difíciles de obtener, por lo tanto, registros derivados de la alimentación en cautiverio proporcionan al menos una aproximación primaria de lo que podrían consumir dentro de su ambiente natural; especialmente cuando la variedad de presas potenciales ofrecidas comparten su misma distribución geográfica y hábitats, y el hecho de que éstas no siempre son reconocidas como alimento (Ayerbe et al., 1990; Henao-Duque y Núñez-Rangel, 2016). Finalmente, la información suministrada aquí aporta alternativas para ser aplicadas en el mantenimiento de estas serpientes en cautiverio, actividad necesaria para la obtención de sus venenos, la base para la producción de sueros antiofídicos y la investigación de sus toxinas (Henao-Duque y Núñez-Rangel, 2016). 


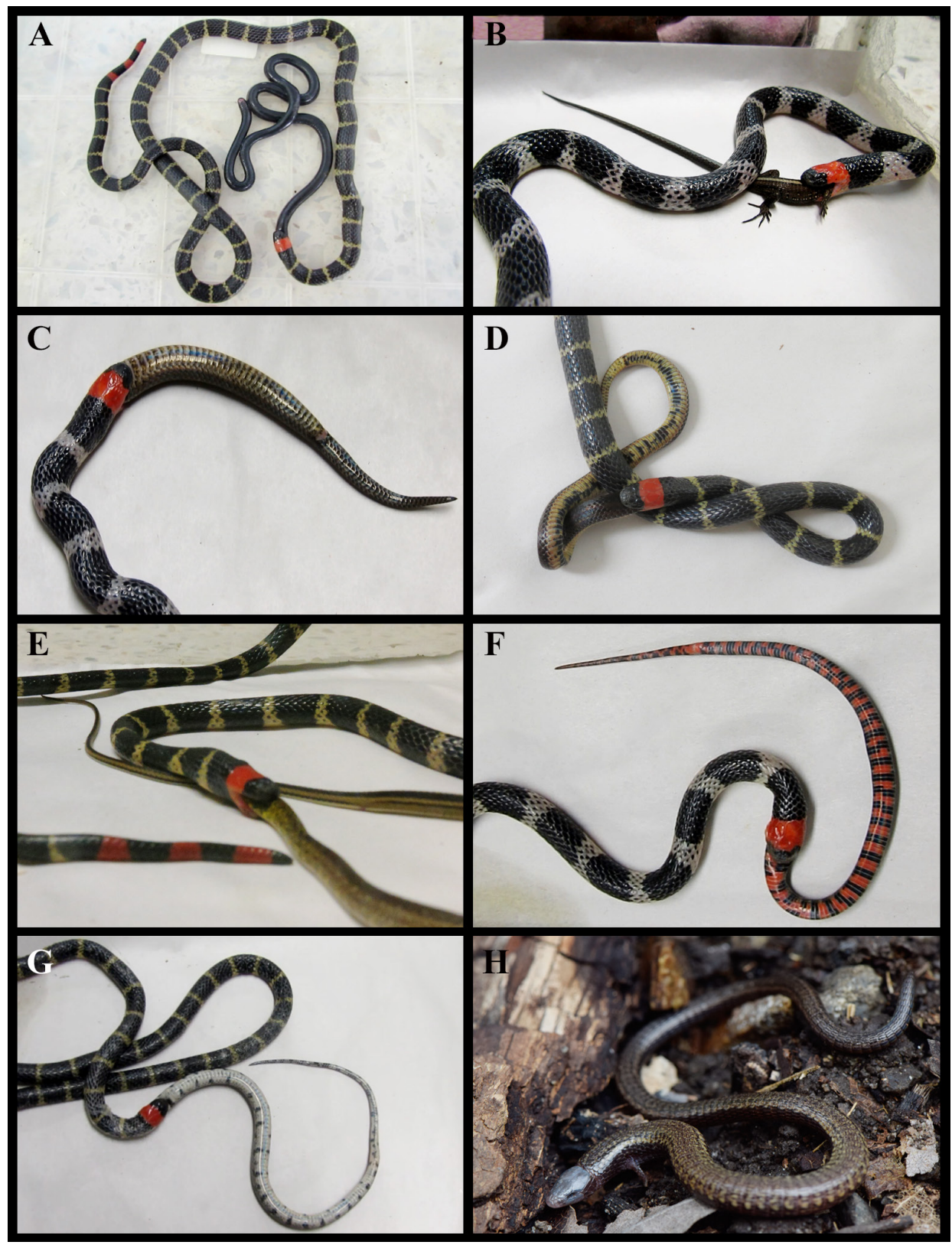

Figura 3. Especies consumidas por Micrurus mipartitus en cautiverio y en su hábitat natural: Caecilia occidentalis (A), Pholidobolus vertebralis (B), Atractus gr. iridescens (C), Atractus lehmanni (D), Dendrophidion bivittatus (E), Erythrolamprus epinephelus (F), Sibon nebulatus $(\mathrm{G})$, Bachia bicolor (H, foto de referencia). M. m. decussatus (A, D, E, G); M. m. popayanensis (B, C, F). 


\section{Agradecimientos}

Al profesor José Toribio Beltrán Vidal por el apoyo incondicional y la confianza que nos brindó durante su dirección en el CIBUC y a Andrés Felipe Liévano Bonilla por su amabilidad y diligencia en los procesos relacionados con la colección herpetológica del Museo de Historia Natural de la Universidad del Cauca. LEV-P y JAZ-B agradecen a Raúl Rodríguez Moreno por la confianza brindada, apoyo incondicional y todo su conocimiento compartido durante las labores de manejo de fauna del PH El Quimbo. Un cálido agradecimiento a Manuel José Romero López por su colaboración en la ardua labor de conseguir alimento para las corales.

\section{Bibliografía}

Ayerbe, S., Tidwell, M. A. y Tidwell, M. 1990. Observaciones sobre la biología y comportamiento de la serpiente coral "rabo de ají” (Micrurus mipartitus): Descripción de una subespecie nueva. Novedades Colombianas Museo de historia natural de la Universidad del Cauca 2: 30-41.

Campbell J. A. y Lamar W. W. 2004. The Venomous Reptiles of the Western Hemisphere. Vol. I. Cornell University Press, Ithaca, New York. 475 pp.

Henao-Duque, A. M. y Núñez-Rangel, V. 2016. Maintenance of red-tail coral snake (Micrurus mipartitus) in captivity and evaluation of individual venom variability. Acta Biológica Colombiana, 21(3): 593-600.

http://www.scielo.org.co/scielo.php?script=sci_arttext \&pid=So120-548X2016000300013

Moreno-Arias, R. y Quintero-Corzo, S. 2015. Reptiles del valle seco del río Magdalena (Huila, Colombia). Caldasia, 37(1): 183-195.

https://revistas.unal.edu.co/index.php/cal/article/view/ 50811 
Pérez-Santos, C. y Moreno, A. G. 1988. Ofidios de Colombia. Museo Regionale di Scienze Naturali, Monografía VI, Torino, Italia. $517 \mathrm{pp}$.

Ríos-Soto, J. A., Arango-Lozano, J. y Rivera-Molina, F. A. 2018. Micrurus mipartitus (Duméril, Bibron \& Duméril, 1854). Pp 37-44. En: Catálogo de Anfibios y Reptiles de Colombia. Universidad de Antioquia, Carmen de Viboral, Antioquia, Colombia. 78 pp.

https://www.researchgate.net/publication/324974812 Micrurus_mipartitus_Dumeril_Bibron_y_Dumeril_1854

Roze, J. A. 1996. Coral snakes of the Americas: Biology, Identification, and Venoms. Krieger Publishing Company, Malabar, Florida, Estados Unidos. 328 pp.

Uetz, P., Freed, P. y Hošek, J. 2019. The Reptile Database. Fecha de acceso: 2019 jul. 23. Disponible en: http://www. reptile-database.org

Wallach, V., Williams, K. L. y Boundy, J. 2014. Snakes of the World: A Catalogue of Living and Extinct Species. Taylor and Francis Group, CRC Press. 1237 pp. 